\title{
Impact of emphysema on sputum culture conversion in male patients with pulmonary tuberculosis: a retrospective analysis
}

Naoki Takasaka ${ }^{1 *}$ D, Yoshitaka Seki ${ }^{1}$, Ikumi Fujisaki ${ }^{1}$, Shota Uchiyama ${ }^{1}$, Sachi Matsubayashi ${ }^{1}$, Akihito Sato ${ }^{1}$, Yumie Yamanaka ${ }^{1}$, Kyuto Odashima ${ }^{1}$, Taisuke Kazuyori ${ }^{1}$, Aya Seki ${ }^{1}$, Hiroshi Takeda', Takeo Ishikawa ${ }^{1}$ and Kazuyoshi Kuwano ${ }^{2}$

\begin{abstract}
Background: Although cigarette smoking may have a negative impact on the clinical outcome of pulmonary tuberculosis (PTB), few studies have investigated the impact of smoking-associated lung diseases. Emphysema is a major pathological finding of smoking-related lung damage. We aimed to clarify the effect of emphysema on sputum culture conversion rate for Mycobacterium tuberculosis (MTB).

Methods: We retrospectively studied 79 male patients with PTB confirmed by acid-fast bacillus smear and culture at Jikei University Daisan Hospital between January 2015 and December 2018. We investigated the sputum culture conversion rates for MTB after starting standard anti-TB treatment in patients with or without emphysema. Emphysema was defined as Goddard score $\geq 1$ based on low attenuation area $<-950$ Hounsfield Unit (HU) using computed tomography (CT). We also evaluated the effect on PTB-related CT findings prior to anti-TB treatment.

Results: Mycobacterial median time to culture conversion (TCC) in 38 PTB patients with emphysema was 52.0 days [interquartile range (IQR) 29.0-66.0 days], which was significantly delayed compared with that in 41 patients without emphysema (28.0 days, IQR 14.0-42.0 days) ( $p<0.001$, log-rank test). Multivariate Cox proportional hazards analysis showed that the following were associated with delayed TCC: emphysema [hazard ratio (HR): $2.43 ; 95 \%$ confidence interval (Cl): 1.18-4.97; $p=0.015)$, cavities (HR: 2.15; 95\% Cl: 1.83-3.89; $p=0.012$ ) and baseline time to TB detection within 2 weeks (HR: $2.95 ; 95 \%$ Cl: 1.64-5.31; $p<0.0001$ ). Cavities and consolidation were more often identified by CT in PTB patients with than without emphysema $(71.05 \%$ vs $43.90 \% ; p=0.015$, and $84.21 \%$ vs $60.98 \% ; p=0.021$, respectively).
\end{abstract}

Conclusions: This study suggests that emphysema poses an increased risk of delayed TCC in PTB. Emphysema detection by CT might be a useful method for prediction of the duration of PTB treatment required for sputum negative conversion.

Keywords: Tuberculosis, Emphysema, Culture conversion

\footnotetext{
* Correspondence: ntakasaka@jikei.ac.jp

'Department of Internal Medicine, Division of Respiratory Diseases, The Jikei University Daisan Hospital, 4-11-1 Izumihoncho Komae-shi, Tokyo 201-8601, Japan

Full list of author information is available at the end of the article
}

(c) The Author(s). 2020 Open Access This article is licensed under a Creative Commons Attribution 4.0 International License, which permits use, sharing, adaptation, distribution and reproduction in any medium or format, as long as you give appropriate credit to the original author(s) and the source, provide a link to the Creative Commons licence, and indicate if changes were made. The images or other third party material in this article are included in the article's Creative Commons licence, unless indicated otherwise in a credit line to the material. If material is not included in the article's Creative Commons licence and your intended use is not permitted by statutory regulation or exceeds the permitted use, you will need to obtain permission directly from the copyright holder. To view a copy of this licence, visit http://creativecommons.org/licenses/by/4.0/ The Creative Commons Public Domain Dedication waiver (http://creativecommons.org/publicdomain/zero/1.0/) applies to the data made available in this article, unless otherwise stated in a credit line to the data. 


\section{Background}

About 10 million people were newly diagnosed with active tuberculosis (TB) and 1.2 million patients died in 2018 [1]. TB clinical features are complicated by bacterial aspects, including drug-resistant Mycobacterium tuberculosis (MTB), and impaired host immune response caused by human immunodeficiency virus (HIV), immunosuppressive therapy, or malignancy [2]. Sputum culture conversion is widely used to determine bacteriological response to anti-TB therapy and is associated with long-term cure [3]. According to recent reports, cigarette smoking is related to delayed culture conversion and treatment failure [4-6]. However, the relationship between actual smoking-related lung damage and pulmonary TB (PTB) is still unknown. Emphysema is a major pathological feature in chronic obstructive pulmonary disease (COPD). The most important aspects of emphysema are sustained oxidative stress and cell damage mediated by macrophages and other cells of the innate and adaptive immune systems, leading to epithelial cell death [7]. Macrophages are localized to sites of alveolar wall destruction in patients with emphysema, and secrete many inflammatory cytokines that contribute to smoking-induced emphysema via elastosis [8]. As a result, there is a correlation between macrophage numbers and severity of emphysema [9]. Alveolar macrophages (AMs) from patients with COPD show reduced phagocytic uptake of bacteria, which might be a factor in bacterial colonization [10].

We have focused on detection of emphysema by computed tomography (CT). Recently, CT biomarkers for emphysema have revealed the relationship between emphysema and COPD phenotype, including airflow limitation and elastic enzyme release [11-14]. Therefore, we hypothesized that CT-detected emphysema might have a negative effect on clinical features of PTB, such as smoking-induced immune dysfunction. To clarify the impact of CT-detected emphysema on culture conversion rates in PTB patients, we performed a retrospective study in our hospital between 2015 and 2018. We showed that CT-detected emphysema had a negative impact on sputum culture conversion in PTB patients.

\section{Methods}

\section{Study design}

In this retrospective study, we analysed data from hospitalized patients with positive acid-fast bacillus (AFB) smears at Jikei University Daisan Hospital between January 2015 and December 2018. During hospitalization, patients maintained smoking cessation. Exclusion criteria were: (1) age < 40 years; (2) isolation of MTB resistant to isoniazid (INH) or rifampicin (RMP); (3) patients not receiving standard anti-TB regimens with $\mathrm{INH}$, RMP, ethambutol (EMB) and pyrazinamide, or INH,
RMP and EMB; (4) patients required desensitization to any adverse effects or comorbidities; (5) co-infection with non-TB mycobacteria; (6) death within 28 days after anti-TB treatment initiation because sputum MTB conversion could not be confirmed; and (7) female sex because only three female PTB patients had emphysema. Sputum smear and culture examinations were performed at the start of treatment and then every 2 weeks, with a margin of error of a few days, during hospitalization. The sputum AFB smear was examined using a fluorochrome procedure according to the American Thoracic Society guidelines [15]. Sputum specimens were cultured in liquid (Mycobacterial Growth Indicator Tubes, MGIT; BD, Franklin Lakes, NJ, USA). Time to sputum culture conversion (TCC) was defined as the number of days between treatment initiation and the first negative sputum culture and no positive culture thereafter. ${ }^{7}$ The delayed conversion may be explained by the high baseline bacillary load. Baseline time to TB detection (TTD) was defined as time (in days) until MGIT culture showed a positive result at initial sputum culture $[6,16]$. Atwine et al. reported a twofold increase in risk of culture conversion at 2 months in patients with baseline TTD $<14$ days compared with $\geq 14$ days [17]. This study was approved by the Ethics Committee of Jikei University School of Medicine [No. 30-003(9024)].

\section{Radiological classification}

Baseline chest high-resolution CT (HRCT) was performed on all patients. HRCT was performed using a helical CT scanner (SOMATOM Definition AS+; Siemens, Erlangen, Germany) with the following parameters: $\quad 1.0-5.0-\mathrm{mm}$ slice thickness, $0.3-\mathrm{mm}$ slice separation, $120-\mathrm{kV}$ tube voltage, $140 \mathrm{mAs}$, $0.6-\mathrm{mm}$ collimation, 0.5 -s rotation time, and 0.85 beam pitch. Images reconstructed with the B60f kernel were used for image analysis. CT scans were requested to establish diagnosis and evaluate active PTB lesions before treatment.

TB-related CT findings were evaluated by considering consolidation, nodule/mass, bronchiolitis and cavities in both lungs $[18,19]$. Consolidation was defined as homogeneous increased lung attenuation that obscured the margin of vessels and airways. A nodule/mass was defined as a well-demarcated spherical area of soft tissue attenuation, $\leq 3 \mathrm{~cm}$ in diameter. Bronchiolitis was defined as the presence of centrilobular micronodules $(<10 \mathrm{~mm}$ in diameter) and branching nodular structures (including tree-in-bud appearance). Cavities were defined as round, gas- or fluid-filled spaces within a nodule/mass or consolidation. CT-detected emphysema was defined as low attenuation area (LAA) $<-950 \mathrm{HU},\left(\mathrm{LAA}_{<-950}\right)$ $[11,20]$. Emphysema severity was assessed according to the Goddard Scoring System [21, 22].: 0, normal; 1, LAA $_{<-950} \leq 25 \%$ affected; $2,>25 \%$ and $\leq 50 \%$ affected; 3 , 
$>50 \%$ and $\leq 75 \%$ affected; and $4,>75 \%$ affected. Six images were analyzed in three slices, which were obtained from the aortic arch level, carina level, and $1 \mathrm{~cm}$ above the right diaphragm, and a total score for six images was calculated for each patient. Two pulmonologists with 11 and 16 years' experience interpreted chest CT results. When there were discrepancies in their readings, final decisions were reached by consensus. Goddard score was determined as the average value scored by the pulmonologists. Total Goddard score $\geq 1$ was defined as emphysema, as reported previously [22], because $95 \%$ of nonsmokers had lungs with $<5 \%$ emphysematous involvement [23].

\section{Statistical analysis}

Statistical analysis was performed using IBM SPSS statistics version 24.0. (IBM Corp., Armonk, NY, USA). Student's $t$ test or Fischer's exact test was used to compare the distributions of characteristics between PTB patients with and without emphysema. Cox proportional hazards regression analysis was used to estimate the hazard ratio (HR) of sputum culture conversion for the following prespecified baseline variables: age, emphysema, smoking history, presence of consolidation, cavity lesions, hypoalbuminemia, and baseline TTD within 2 weeks. HRs were calculated with 95\% confidence intervals (CIs). A $p$ value $<0.05$ indicated statistical significance in all analyses. Kaplan-Meier curves for TB sputum conversion probability were plotted for patients with emphysema versus those without emphysema. The log-rank test was used to test the significance in subgroups of patients with emphysema.

\section{Results}

\section{Patient characteristics}

Between January 2015 and December 2018, 171 patients were diagnosed and hospitalized with active TB with
AFB smear- and culture-positive sputum at Jikei Daisan Hospital (Fig. 1). Ninety-two patients were excluded according to the exclusion criteria. The mean age was $69.01 \pm 15.92$ years. No patient was positive for HIV antibody. There was no significant difference in mean age, proportion of patients with chronic kidney disease or diabetes, or baseline TTD between patients with or without emphysema (Table 1). PTB patients with emphysema had a significantly higher proportion with smoking history (100\% vs $41.46 \%, p<0.0001)$, current smoking habit $(52.63 \%$ vs $14.63 \%, p<0.001)$, and hypoalbuminemia ( $81.58 \%$ vs $46.34 \%, p=0.001$ ) (Table 1$)$. About a quantitative assessment of emphysema, average Goddard score of emphysema patients was $6.74 \pm 4.73$ (Table 1, Fig. 2).

\section{CT findings related to PTB}

We compared PTB-related CT findings including cavities, consolidation, bronchiolitis, and nodule/mass between PTB patients with or without emphysema. Cavities and consolidation were seen more frequently in PTB patients with than without emphysema $(71.05 \%$ vs $43.90 \%, p=0.015$, and $84.21 \%$ vs $60.98 \% ; p=0.021$, respectively) (Table 2).

\section{Sputum TCC}

In the Kaplan-Meier analysis, 38 PTB patients with emphysema needed a median TCC of 52.0 days [interquartile range (IQR) 29-66 days], which was significantly longer than 28.0 days (IQR 14-42 days) in 41 patients without emphysema $(p<0.001$, log-rank test) (Fig. 3). Univariate Cox proportional hazards regression analysis showed that the following were associated with delayed TCC: emphysema (HR: 2.21; 95\% CI: 1.36-3.60; $p=$ 0.001 ); smoking history (HR: 1.81; 95\% CI: 1.09-2.99; $p=0.021$ ); consolidation (HR: 1.77 ; $95 \% \mathrm{CI}: 1.06-2.96$;

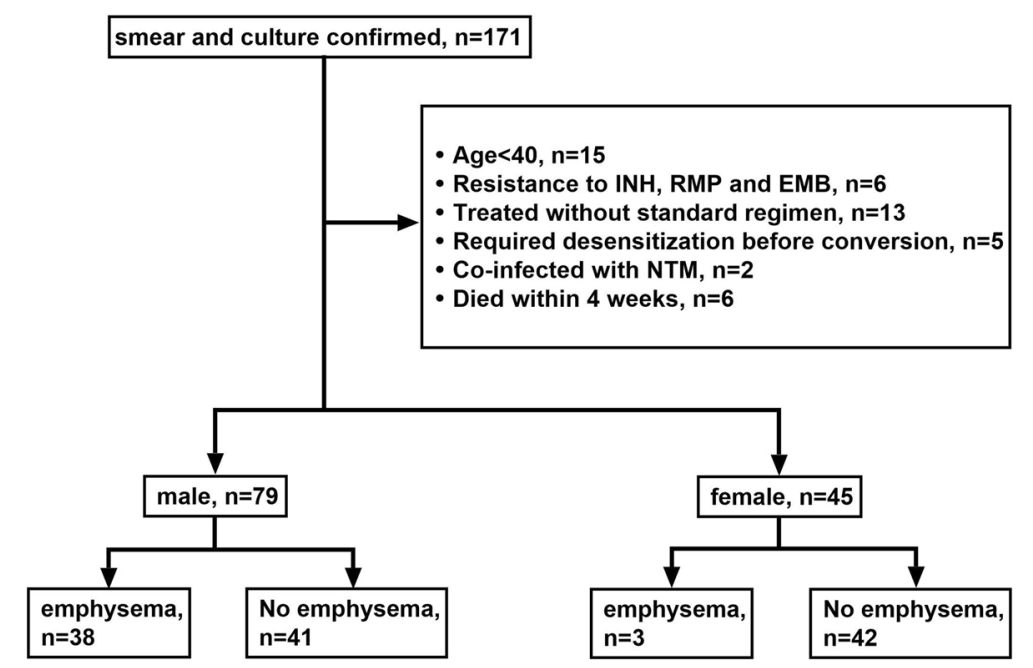

Fig. 1 Flow chart of patient selection. EMB, ethambutol; INH, isoniazid; NTM, nontuberculous mycobacteria; RMP, rifampicin 
Table 1 Characteristics of patients with pulmonary tuberculosis with or without emphysema

\begin{tabular}{|c|c|c|c|c|}
\hline Characteristics & $\begin{array}{l}\text { Total } \\
(n=79)\end{array}$ & With emphysema $(n=38)$ & $\begin{array}{l}\text { Without emphysema } \\
(n=41)\end{array}$ & $p$-value ${ }^{*}$ \\
\hline \multicolumn{5}{|l|}{ Demographics } \\
\hline Age, years & $69.01 \pm 15.92$ & $69.18 \pm 16.35$ & $68.85 \pm 15.72$ & 0.927 \\
\hline Smoking history & $55(69.62 \%)$ & $38(100 \%)$ & $17(41.46 \%)$ & $<0.0001$ \\
\hline Current smoker & $26(32.91 \%)$ & $20(52.63 \%)$ & $6(14.63 \%)$ & $<0.001$ \\
\hline Alcohol consumption & $27(34.18 \%)$ & $17(44.74 \%)$ & $10(24.39 \%)$ & 0.057 \\
\hline AST (IU/L) & $29.29 \pm 25.14$ & $33.13 \pm 31.65$ & $25.73 \pm 16.69$ & 0.193 \\
\hline ALT (IU/L) & $21.58 \pm 21.32$ & $24.03 \pm 26.06$ & $19.32 \pm 15.73$ & 0.330 \\
\hline Total bilirubin (mg/dL) & $0.55 \pm 0.35$ & $0.45 \pm 0.20$ & $0.64 \pm 0.43$ & 0.011 \\
\hline Diabetes $^{\dagger}$ & $22(27.85 \%)$ & $13(34.21 \%)$ & $9(21.95 \%)$ & 0.225 \\
\hline $\begin{array}{l}\text { Haemoglobin A1c (\%) } \\
\text { (missing value = 1) }\end{array}$ & $6.42 \pm 1.50$ & $6.40 \pm 1.26$ & $6.43 \pm 1.71$ & 0.930 \\
\hline$C K D^{\ddagger}$ & 19 (24.05\%) & $7(18.42 \%)$ & $12(29.27 \%)$ & 0.260 \\
\hline eGFR (mL/min/1.73 m²) & $77.94 \pm 40.36$ & $79.37 \pm 30.10$ & $76.61 \pm 48.33$ & 0.764 \\
\hline Hypoalbuminemia ${ }^{\S}$ & $50(63.29 \%)$ & $31(81.58 \%)$ & $19(46.34 \%)$ & 0.001 \\
\hline Albumin (g/dL) & $3.14 \pm 0.76$ & $2.93 \pm 0.68$ & $3.33 \pm 0.80$ & 0.020 \\
\hline Autoimmunity & $5(6.33 \%)$ & $1(2.63 \%)$ & $4(9.76 \%)$ & 0.361 \\
\hline Steroid use & $8(10.13 \%)$ & $2(5.26 \%)$ & $6(14.63 \%)$ & 0.266 \\
\hline Malignancy & $9(11.39 \%)$ & $4(10.53 \%)$ & $5(12.20 \%)$ & 1.0 \\
\hline CRP (mg/dL) & $5.08 \pm 4.35$ & $5.58 \pm 3.61$ & $4.60 \pm 4.95$ & 0.320 \\
\hline TTD at baseline (days) & $17.19 \pm 7.07$ & $17.13 \pm 7.75$ & $17.24 \pm 6.47$ & 0.944 \\
\hline TTD within 2 weeks at baseline & $54(68.35 \%)$ & $25(65.79 \%)$ & $29(70.73 \%)$ & 0.637 \\
\hline Treated with HREZ & $54(68.35 \%)$ & $27(71.05 \%)$ & $27(65.85 \%)$ & 0.620 \\
\hline Goddard score & $3.24 \pm 4.70$ & $6.74 \pm 4.73$ & 0 & $<0.0001$ \\
\hline
\end{tabular}

Data are means \pm SD, $\mathrm{n}(\%)$

*We used Fisher's exact test or Student's t-test to calculate $p$-values

${ }^{\dagger}$ Diabetes: prescription of oral hypoglycaemic agents and/or insulin; fasting blood glucose $\geq 126 \mathrm{mg} / \mathrm{dL}$ or haemoglobin A1c $\geq 6.5 \%$. ${ }^{\ddagger} \mathrm{CKD}$ : glomerular filtration rate $<60 \mathrm{~mL} / \mathrm{min} / 1.73 \mathrm{~m} 2$ for $>3$ months. ${ }^{\S}$ Hypoalbuminemia: serum albumin $\leq 3.5 \mathrm{~g} / \mathrm{dL}$

$S D$ standard deviation, CKD chronic kidney disease, TTD time to tuberculosis detection, HREZ isoniazid, rifampicin, ethambutol and pyrazinamide

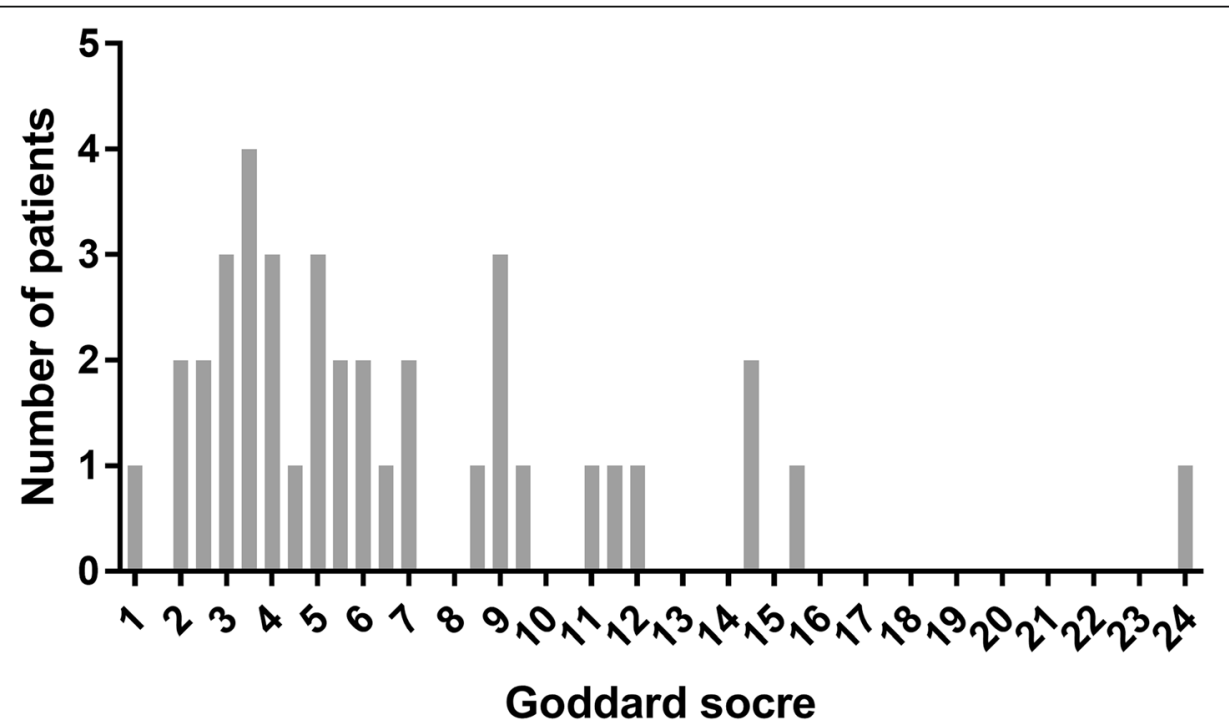

Fig. 2 The distribution of Goddard score in the pulmonary tuberculosis patients with emphysema $(n=38)$ 
Table $\mathbf{2} C T$ findings in patients with pulmonary tuberculosis with or without emphysema

\begin{tabular}{lllll}
\hline CT findings & $\begin{array}{l}\text { Total } \\
\mathbf{n = 7 9}\end{array}$ & $\begin{array}{l}\text { With emphysema } \\
\mathbf{n = 3 8}\end{array}$ & $\begin{array}{l}\text { Without emphysema } \\
\mathbf{n = 4 1}\end{array}$ & $\boldsymbol{p}$ - value* \\
\hline Cavities & $45(56.96 \%)$ & $27(71.05 \%)$ & $18(43.90 \%)$ & 0.015 \\
Consolidation & $57(72.15 \%)$ & $32(84.21 \%)$ & $25(60.98 \%)$ & 0.021 \\
Bronchiolitis & $62(78.48 \%)$ & $28(73.68 \%)$ & $34(82.93 \%)$ & 0.318 \\
Nodule/mass & $29(36.71 \%)$ & $16(42.11 \%)$ & $13(31.71 \%)$ & 0.338 \\
\hline
\end{tabular}

Data are $\mathrm{n}(\%)$

*We used Fisher's exact test

$p=0.031$ ); cavities (HR: 2.72; 95\% CI: $1.65-4.49 ; p<$ 0.0001); hypoalbuminemia (HR: $1.74 ; 95 \%$ CI: $1.08-2.80$; $p=0.024$ ); and baseline TTD within 2 weeks (HR: 2.56; 95\% CI: $1.52-4.31 ; p<0.0001$ ) (Table 3). Multivariate Cox proportional hazards regression analysis showed that the following were associated with delayed TCC after adjusting for age, emphysema, smoking history, cavities, consolidation, hypoalbuminemia, and baseline TTD within 2 weeks: emphysema (HR: $2.43 ; 95 \%$ CI: $1.18-4.97$; $p=0.015$ ); cavities (HR: 2.15 ; $95 \% \mathrm{CI}: 1.18-$ 3.89; $p=0.012$ ); and baseline TTD within 2 weeks (HR: 2.95; 95\% CI: 1.64-5.31; p < 0.0001) (Table 3).

\section{Discussion}

We evaluated the impact of emphysema on AFB sputum TCC rates following initiation of anti-TB treatment in hospitalized PTB patients at Jikei University Daisan Hospital. Median TCC was significantly delayed in 38 PTB patients (52.0 days) with emphysema compared with 41 patients without emphysema (28.0 days). Multivariate Cox proportional hazards analysis showed that emphysema, cavities, and baseline TTD within 2 weeks were associated with delayed TCC. With regard to PTB-related CT findings, cavities and consolidation were seen more frequently in PTB patients with than without emphysema $(71.05 \%$ vs $43.90 \% ; p=0.015$, and $84.21 \%$ vs $60.98 \% ; p=0.021$, respectively).
To our knowledge, this is the first study to report the impact of emphysema on sputum culture conversion in PTB patients. We focused on emphysema detection using CT. Emphysema was defined using the current standard of $\mathrm{LAA}_{<-950}[11,14]$. Although no wellvalidated biomarker of COPD has been identified other than forced expiratory volume in $1 \mathrm{~s}\left(\mathrm{FEV}_{1}\right)$, various quantitative imaging methods for emphysema are being developed to reveal the relationship between emphysema and COPD phenotype, including airflow limitation and level of matrix metalloproteinases (MMPs) [11-14]. CT is a minimally invasive technique that can identify the pathological features of emphysema. Goddard score based on the LAA proportion is widely used to measure emphysema [22, 24, 25]. Average Goddard score of emphysema patients was $6.74 \pm 4.73$ in our study. The severity of most emphysema cases was categorised as mild or moderate according to previous reports [26]. Our data suggest that even a mild level of emphysema has a negative influence on the duration of PTB treatment required for sputum culture conversion. Multivariate Cox proportional hazards analysis showed that emphysema was more significantly associated with delayed MTB culture conversion than smoking history was (not significant). According to recent reports, smoking history (current or former) has a negative impact on PTB clinical outcome, including delayed culture conversion [6, 27]. Although smoking history based on medical interview is a simple method to

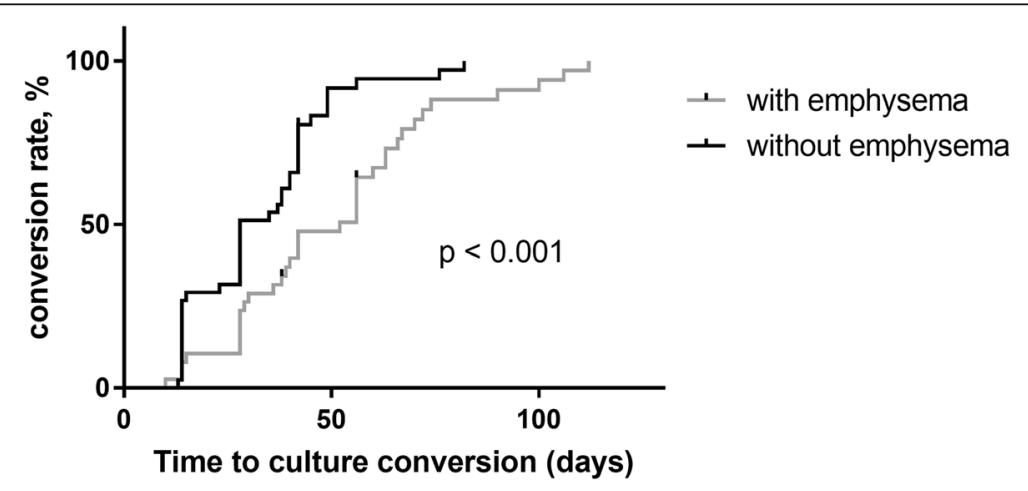

Fig. 3 Proportion of pulmonary tuberculosis (PTB) patients with sputum culture conversion after treatment initiation (with vs without emphysema). Kaplan-Meier curves revealed significantly longer sputum time to culture conversion (TTC) in 38 PTB patients with emphysema (median TCC 52.0 days) than in 41 patients without emphysema (28.0 days) ( $p<0.001$, log-rank test) 
Table 3 Cox proportional hazard regression unadjusted and adjusted analysis of time to sputum culture conversion*

\begin{tabular}{|c|c|c|c|c|}
\hline Characteristics & Univariate HR (95\% Cl) & $p$-value & Multivariate HR $(95 \% \mathrm{Cl})$ & $p$-value \\
\hline Age & $1.01(0.99-1.02)$ & 0.483 & $0.994(0.98-1.01)$ & 0.593 \\
\hline Emphysema & $2.21(1.36-3.60)$ & 0.001 & $2.43(1.18-4.97)$ & 0.015 \\
\hline Smoking history & $1.81(1.09-2.99)$ & 0.021 & $0.87(0.44-1.72)$ & 0.692 \\
\hline Current smoker & $1.26(0.77-2.04)$ & 0.361 & & \\
\hline Consolidation & $1.77(1.06-2.96)$ & 0.031 & $1.07(0.61-1.87)$ & 0.818 \\
\hline Cavities & $2.72(1.65-4.49)$ & $<0.0001$ & $2.15(1.18-3.89)$ & 0.012 \\
\hline Alcohol consumption & $1.19(0.73-1.94)$ & 0.478 & & \\
\hline Diabetes $^{\dagger}$ & $1.67(0.99-2.82)$ & 0.055 & & \\
\hline$C K D^{\ddagger}$ & $0.83(0.48-1.41)$ & 0.482 & & \\
\hline Hypoalbuminemia $^{\S}$ & $1.74(1.08-2.80)$ & 0.024 & $0.99(0.53-1.86)$ & 0.979 \\
\hline Autoimmunity & $0.44(0.18-1.11)$ & 0.081 & & \\
\hline Steroid use & $0.83(0.38-1.82)$ & 0.641 & & \\
\hline Malignancy & $1.02(0.51-2.06)$ & 0.956 & & \\
\hline TTD within 2 weeks at baseline & $2.56(1.52-4.31)$ & $<0.0001$ & $2.95(1.64-5.31)$ & $<0.0001$ \\
\hline Treated with HREZ & $1.40(0.85-2.31)$ & 0.181 & & \\
\hline
\end{tabular}

* HR calculated with each per unit increase. Each variable measured at the time of diagnosis. Adjusted by previously described clinically important variables and candidate variables (age, emphysema, smoking history, consolidation, cavities, hypoalbuminemia and TTD within 2 weeks) for which the $p$ - value was < 0.05 on univariate analysis

${ }^{\dagger}$ Diabetes: prescription of oral hypoglycaemic agents and/or insulin; fasting blood glucose $\geq 126 \mathrm{mg} / \mathrm{dL}$ or haemoglobin A1C $\geq 6.5 \%$. ${ }^{\ddagger}$ CKD: glomerular filtration rate $<60 \mathrm{~mL} / \mathrm{min} / 1.73 \mathrm{~m} 2$ for $>3$ months. ${ }^{\S}$ Hypoalbuminemia: serum albumin $\leq 3.5 \mathrm{~g} / \mathrm{dL}$

assess smoking exposure, the relationship between amount of smoking and smoking-associated immune response remains unclear. The reversibility of immune modification by smoking cessation is still unknown. Our results suggest that emphysema detection using CT is useful for predicting the duration of TB treatment required for sputum culture negative conversion.

The host immune response is crucial in TB pathogenesis, as demonstrated by the increase in incidence and mortality in immunocompromised hosts [2]. AMs are involved in the host inflammatory defence against MTB [28]. MTB is transmitted through air droplets and is an intracellular bacterium that infects AMs and dendritic cells (DCs) localized to the alveoli. The innate immune response is activated and inflammatory cells are recruited to the lungs. MTB that can evade the host immune system within AMs and DCs drives granulomatous inflammation and establishes PTB.

Recent reports suggest that smoking habit increases the risk of developing active TB and mortality [27, 29], and smoking-modulated immune dysfunction might be involved in TB pathogenesis $[6,27]$. Although the pathogenesis of COPD/emphysema is not fully understood, it is associated with smoking-induced chronic inflammation of airways and lung parenchyma. After amplification of inflammation in patients with COPD, abnormal inflammatory immune responses are sustained even after smoking cessation [30]. AMs also play a central role in chronic inflammation in COPD [9]. AMs are activated by cigarette smoke extract, and secrete inflammatory mediators, including tumour necrosis factor- $\alpha$, chemokine $\mathrm{C}-\mathrm{X}-\mathrm{C}$ motif ligand (CXCL)1 and CXCL8. AMs release elastic enzymes (MMPs) that contribute to emphysematous changes [31]. Macrophages are localized to sites of alveolar wall destruction in patients with emphysema, and there is a correlation between number of AMs and severity of emphysema [9]. AMs from patients with COPD show reduced phagocytic uptake of bacteria, which might be a factor in bacterial colonization [10, 29]. These reports suggest that smoking-associated immune modification in emphysema is involved in delayed TCC in terms of increased PTB severity and reduced response to anti-TB therapy.

Patients' immune function also affects the radiological findings of PTB [32]. PTB-related CT findings differ between patients with or without emphysema. Jeon et al. reported that PTB patients with emphysema often show pneumonia-like consolidation, suggesting that impaired innate immunity in emphysema causes PTB progression and expansion of inflammation [18]. In our study, cavities and consolidation were seen more frequently in PTB patients with emphysema than in those without emphysema (71.05\% vs $43.90 \% ; p=0.015$, and $84.21 \%$ vs $60.98 \% ; p=0.021$, respectively) (Table 2). Cavity lesions are associated with the greatest bacterial load, suggesting highly infectious and severe PTB [33, 34]. It is speculated that smoking-related immune modification in emphysema contributes to more severe radiological findings and extension of TCC.

This study had some limitations. First, this was a small, single-center retrospective study. Nevertheless, our finding 
of a negative effect of emphysema on sputum TCC suggests that emphysema is a potential risk factor for PTB development in terms of smoking-related immune dysfunction. In future, a larger prospective study could reveal the relationship between CT-detected emphysema and clinical outcome of PTB. Second, we needed to enroll patients with confirmed COPD with emphysema to determine the relationship between COPD/emphysema and sputum culture conversion. To diagnose COPD, airflow limitation must be identified with spirometry. However, it is impossible to estimate accurately airflow limitation in patients with COPD and active PTB at baseline.

Third, we could not analyze female patients because there were only three women with PTB with emphysema. From the 1970s to 1990s, smoking rate for Japanese women was only $10-15 \%$, while that for men was $55-80 \%$ [35]. Although the difference became smaller after the 2000s, the risk of emphysema in Japanese women is potentially lower than in men.

\section{Conclusions}

In conclusion, CT-detected emphysema with a Goddard score $\geq 1$ is associated with delayed AFB sputum culture conversion and PTB-related CT findings. If confirmed in larger populations of PTB patients with emphysema, CT-detected emphysema could be helpful for predicting the duration of PTB treatment required for sputum culture negative conversion.

\section{Abbreviations}

PTB: Pulmonary tuberculosis; MTB: Mycobacterium tuberculosis;

TB: Tuberculosis; HU: Hounsfield Unit; CT: Computed tomography; TCC: Time to culture conversion; IQR: Interquartile range; HR: Hazard ratio;

$\mathrm{Cl}$ : Confidence interval; HIV: Human immunodeficiency virus; COPD: Chronic obstructive pulmonary disease; AM: Alveolar macrophage; AFB: Acid-fast bacillus; INH: Isoniazid; RMP: Rifampicin; EMB: Ethambutol;

MGIT: Mycobacterial Growth Indicator Tubes; TTD: Time to TB detection; HRCT: High-resolution computed tomography; LAA: Low attenuation area; SD: Standard deviation; CKD: Chronic kidney disease; HREZ: Isoniazid, rifampicin, ethambutol and pyrazinamide.; FEV ${ }_{1}$ : Forced expiratory volume in 1 s; MMP: Matrix metalloproteinase; DC: Dendritic cell; CXCL: Chemokine (C$\mathrm{X}-\mathrm{C}$ motif) ligand

\section{Acknowledgments}

We thank Cathel Kerr, BSc, PhD, from Edanz Group (https://en-authorservices.edanzgroup.com/) for editing a draft of this manuscript.

\section{Authors' contributions}

NT conceived the study. NT, YS and KK designed the study. NT and IF reviewed chest $C T$ findings. NT and YS wrote the manuscript. NT, YS, SU, SM, AS, YY, KO, TK, AS, HT and TI contributed to data collection and data analysis. All authors read and approved the final manuscript.

\section{Funding}

None.

\section{Availability of data and materials}

The datasets used and/or analyses during the current study available from the corresponding author on reasonable request.

\section{Ethics approval and consent to participate}

In the retrospective study, written informed consent from the participants involved was unavailable and unnecessary. The present study was carried out by opt-out consent, and the information about opting out was on a bulletin board in our hospital. This study was approved by the Ethics Committee of Jikei University School of Medicine [No. 30-003(9024)].

\section{Consent for publication}

Not applicable.

\section{Competing interests}

The authors declare that they have no competing interests.

\section{Author details}

'Department of Internal Medicine, Division of Respiratory Diseases, The Jikei University Daisan Hospital, 4-11-1 Izumihoncho Komae-shi, Tokyo 201-8601, Japan. ${ }^{2}$ Department of Internal Medicine, Division of Respiratory Diseases, The Jikei University School of Medicine, Tokyo, Japan.

Received: 9 July 2020 Accepted: 27 October 2020

Published online: 07 November 2020

\section{References}

1. World Health Organization. Global tuberculosis report 2019. https://www. who.int/tb/publications/global_report/en/. Accessed 8 July 2020.

2. World Health Organization. WHO Guidelines on tuberculosis infection prevention and control, 2019 update. https://www.who.int/tb/publications/2 019/guidelines-tuberculosis-infection-prevention-2019/en/. Accessed 8 July 2020.

3. Mitchison DA. Assessment of new sterilizing drugs for treating pulmonary tuberculosis by culture at 2 months. Am Rev Respir Dis. 1993;147:1062-3.

4. Kanda R, Nagao T, Tho NV, et al. Factors affecting time to sputum culture conversion in adults with pulmonary tuberculosis: a historical cohort study without censored cases. PLoS One. 2015;10:e0142607.

5. Hung C-L, Su P-L, Ou C-Y. Prognostic effect of tuberculosis on patients with occupational lung diseases. Medicine. 2016;95:e4748.

6. Reimann M, Schaub D, Kalsdorf B, et al. Cigarette smoking and culture conversion in patients with susceptible and M/XDR-TB. Int J Tuberc Lung Dis. 2019;23:93-8

7. Craig JM, Scott AL, Mitzner W. Immune-mediated inflammation in the pathogenesis of emphysema: insights from mouse models. Cell Tissue Res. 2017:367:591-605.

8. Byrne AJ, Mathie SA, Gregory LG, et al. Pulmonary macrophages: key players in the innate defence of the airways. Thorax. 2015;70:1189-96.

9. Barnes PJ. Inflammatory mechanisms in patients with chronic obstructive pulmonary disease. J Allergy Clin Immunol. 2016;138:16-27.

10. Taylor AE, Finney-Hayward TK, Quint JK, et al. Defective macrophage phagocytosis of bacteria in COPD. Eur Respir J. 2010;35:1039-47.

11. Galbán CJ, Han MK, Boes JL, et al. Computed tomography-based biomarker provides unique signature for diagnosis of COPD phenotypes and disease progression. Nat Med. 2012;18:1711-5.

12. Ostridge K, Williams N, Kim V, et al. Distinct emphysema subtypes defined by quantitative $C T$ analysis are associated with specific pulmonary matrix metalloproteinases. Respir Res. 2016;17:92.

13. Castaldi PJ, Estépar RSJ, Mendoza CS, et al. Distinct quantitative computed tomography emphysema patterns are associated with physiology and function in smokers. Am J Respir Crit Care Med. 2013;188:1083-90.

14. Smith BM, Austin JH, Newell JD, et al. Pulmonary emphysema subtypes on computed tomography: the MESA COPD study. Am J Med. 2014;127:94.e7-23.

15. Diagnostic Standards and Classification of Tuberculosis in Adults and Children. Am J Respir Crit Care Med. 2012;161:1376-95.

16. Dorman SE, Johnson JL, Goldberg S, et al. Substitution of moxifloxacin for isoniazid during intensive phase treatment of pulmonary tuberculosis. Am J Respir Crit Care Med. 2009;180:273-80.

17. Atwine D, Orikiriza P, Taremwa I, et al. Predictors of delayed culture conversion among Ugandan patients. BMC Infect Dis. 2017;17:299.

18. Jeon $\mathrm{KN}, \mathrm{Ha} \mathrm{JY}$, Park MJ, et al. Pulmonary tuberculosis in patients with emphysema. J Comput Assist Tomogr. 2016;40:912-6.

19. Song JW, Koh W-JJ, Lee KS, et al. High-resolution CT findings of Mycobacterium avium-intracellulare complex pulmonary disease: correlation with pulmonary function test results. AJR Am J Roentgenol. 2008;191:W160. 
20. Gevenois PA, de Maertelaer V, Vuyst PD, et al. Comparison of computed density and macroscopic morphometry in pulmonary emphysema. Am J Respir Crit Care Med. 1995;152:653-7.

21. Goddard PR, Nicholson EM, Laszlo G, et al. Computed tomography in pulmonary emphysema. Clin Radiol. 1982;33:379-87.

22. Makino $Y$, Shimada $Y$, Hagiwara M, et al. Assessment of emphysema severity as measured on three-dimensional computed tomography images for predicting respiratory complications after lung surgery. Eur I Cardiothorac Surg. 2018;54:671-6.

23. Ueda K, Jinbo M, Li T-SS, et al. Computed tomography-diagnosed emphysema, not airway obstruction, is associated with the prognostic outcome of early-stage lung cancer. Clin Cancer Res. 2006;12:6730-6.

24. Lee HY, Kim EY, Kim YS, et al. Prognostic significance of CT-determined emphysema in patients with small cell lung cancer. J Thorac Dis. 2018;10:874-81.

25. Kim Y, Kim E, Ahn H, et al. Prognostic significance of CT-emphysema score in patients with advanced squamous cell lung cancer. J Thorac Dis. 2016;8: 1966-73.

26. Makita H, Nasuhara Y, Nagai K, et al. Characterisation of phenotypes based on severity of emphysema in chronic obstructive pulmonary disease. Thorax. 2007;62:932-7.

27. Lin $\mathrm{H}-\mathrm{HH}$, Ezzati $\mathrm{M}$, Chang $\mathrm{H}-\mathrm{YY}$, et al. Association between tobacco smoking and active tuberculosis in Taiwan: prospective cohort study. Am J Respir Crit Care Med. 2009;180:475-80.

28. van Crevel R, Ottenhoff TH, van der Meer JW. Innate immunity to Mycobacterium tuberculosis. Clin Microbiol Rev. 2002;15:294-309.

29. Inghammar M, Ekbom A, Engström G, et al. COPD and the risk of tuberculosis--a population-based cohort study. PLoS One. 2010;5:e10138.

30. Brusselle GG, Joos GF, Bracke KR. New insights into the immunology of chronic obstructive pulmonary disease. Lancet. 2011;378:1015-26.

31. Russell RE, Thorley A, Culpitt SV, et al. Alveolar macrophage-mediated elastolysis: roles of matrix metalloproteinases, cysteine, and serine proteases. Am J Physiol Lung Cell Mol Physiol. 2002;283:L867-73.

32. Jones $B E$, Ryu $R$, Yang $Z$, et al. Chest radiographic findings in patients with tuberculosis with recent or remote infection. Am J Respir Crit Care Med. 1997;156:1270-3.

33. Canetti G. Present aspects of bacterial resistance in tuberculosis. Am Rev Respir Dis. 1965;92:687-703.

34. Kosaka N, Sakai T, Uematsu H, et al. Specific high-resolution computed tomography findings associated with sputum smear-positive pulmonary tuberculosis. J Comput Assist Tomogr. 2005;29:801-4.

35. JAPAN HEALTH PROMOTION \& FITNESS FOUNDATION. Tobacco or health http://www.health-net.or.jp/tobacco/product/pd090000.html. Accessed 8 July 2020

\section{Publisher's Note}

Springer Nature remains neutral with regard to jurisdictional claims in published maps and institutional affiliations.

\section{Ready to submit your research? Choose BMC and benefit from:}

- fast, convenient online submission

- thorough peer review by experienced researchers in your field

- rapid publication on acceptance

- support for research data, including large and complex data types

- gold Open Access which fosters wider collaboration and increased citations

- maximum visibility for your research: over $100 \mathrm{M}$ website views per year

At $\mathrm{BMC}$, research is always in progress.

Learn more biomedcentral.com/submissions 